\title{
Análise quantitativa da desmineralização do esmalte dental submetido à ação de dentifrícios fluoretados
}

\author{
Elisângela de Jesus Campos ${ }^{1}$ \\ Roberto Paulo Correia de Araújo \\ Maria Thereza Barral Araújo \\ Danilo Barral Araújo ${ }^{3}$ \\ Max José Pimenta Lima ${ }^{4}$ \\ Nayara de Sá Oliveira \\ Alexnaldo Correia Dias ${ }^{3}$
}

\section{Resumo}

A cárie dental é uma doença multifatorial, caracterizada como um processo dinâmico, originado pelo desequilíbrio nos fenômenos de desmineralização e remineralização. Diversos estudos têm demonstrado que a prevalência de cárie, na maioria dos países, diminuiu nas últimas duas décadas, devido à implementação de programas de saúde pública, ao uso do flúor através da fluoretação das águas de abastecimento e o uso de dentifrícios fluoretados. O flúor contido nos dentifrícios é capaz de reduzir a perda mineral do esmalte dos dentes hígidos e ativar a reposição iônica em unidades com lesôes de manchas brancas e com lesôes incipientes de cárie. Esses dados justificam o presente estudo, que teve por objetivo determinar, in vitro, o grau de proteção do esmalte dentário humano pelo flúor, nas formas de fluoreto de sódio $(\mathrm{NaF})$ e monofluorfosfato de sódio (MFP), contidos em sete dentifrícios-teste (Colgate Proteção Anticáries (DT), Trends $\left(\mathrm{DT}_{2}\right.$ ), Prevent Antiplaca (DT $)$, Colgate Antitártaro $\left(\mathrm{DT}_{4}\right)$, Phillips 2 com Flúor $\left(\mathrm{DT}_{5}\right)$, Gessy Cristal Juá e Hortelã $\left(\mathrm{DT}_{6}\right)$ e Colgate Proteção Anticáries $\mathrm{Gel}\left(\mathrm{DT}_{7}\right)$, comparativamente à ação do dentifrício-controle, sem fluoreto, Phillips (DC), frente à queda do $\mathrm{pH}$ aos níveis críticos. As taxas do cálcio e do fosfato liberados do esmalte, previamente tratado pelos dentifrícios, foram determinadas através da técnica de espectrometria de emissão atômica, com plasma indutivamente acoplado. Os resultados encontrados revelaram o poder protetor desses produtos, indicando o adequado controle da qualidade industrial. Constatou-se, também, maior proteção do esmalte pelos dentifrícios que contêm fluoreto de sódio, em relação àqueles que continham monofluorfosfato de sódio, e uma correlação negativa entre as taxas do cálcio e do fosfato liberados do esmalte dental humano.

Palavras-chave: Esmalte dental - Dentifrícios; fluoreto de sódio (NaF); monofluorfosfato de sódio (MFP).

\section{INTRODUÇÃO}

A escovação dental é um procedimento universalmente, indicado com a finalidade de remover o acúmulo e controlar a formação do biofilme supragengival. Com o objetivo de facilitar, tecnicamente, sua execução, vêm sendo introduzidos no mercado diversos modelos de

\footnotetext{
${ }^{1}$ Professora Assistente de Bioquímica. Faculdade de Odontologia. FBDC. Salvador - BA

${ }^{2}$ Professor Adjunto de Bioquímica. Instituto de Ciências da Saúde. UFBA. Salvador - BA

${ }^{3}$ Acadêmico do Curso de Odontologia. UFBA. Salvador - BA

${ }^{4}$ Professor Assistente de Bioquímica. Instituto de Ciências Biológicas. UCSAL. Salvador - BA

${ }^{5}$ Professora Substituta de Bioquímica. Instituto de Ciências da Saúde. UFBA. Salvador - BA
}

Correspondência para / Correspondence to:

Roberto Paulo Correia de Araújo.

Laboratório de Bioquímica Oral-Departamento de Biofunção- ICS - UFBA.

Av. Reitor Miguel Calmon, s/n ${ }^{\circ}$ - Campus do Canela.

40.110-100 Salvador - Bahia - Brasil.

Tel.: (71) 3245-8602. Fax: (71) 32458917.

E-mail: rpcaraujo@hotmail.com 
escovas dentais com diferentes tamanhos, formatos e dureza de cerdas. Associados às técnicas convencionais de escovação, os dentifrícios são as formulações químicas de prevenção odontológica mais utilizadas pela população. De variadas procedências industriais, contêm diversos componentes ativos que contribuem para o controle do supracitado biofilme. (PALOMO et al.,1989)

Cada componente tem a função de assegurar aos dentifrícios em geral os efeitos cosméticos e preventivo-terapêuticos desejados, dentre os quais o abrasivo, o flavorizante, o detergente e o farmacológico, a depender da indicação clínica. Do ponto de vista farmacotécnico, determinados componentes garantem relevantes propriedades físicas às diversas formulações, tais como os agentes aglutinantes, utilizados para garantir a homogeneidade da formulação, como o carboximetilcelulose; os umectantes, fundamentais para impedir a desidratação e o ressecamento dos cremes dentais nas embalagens, como o sorbitol e o glicerol, assim como os conservantes, utilizados para preservar o dentifrício da contaminação bacteriana, como o phidroxibenzoato de metila. ( CURY, 2002)

$\mathrm{O}$ uso inadequado e excessivo do conjunto escova dental e dentifrício tem a possibilidade de ocasionar lesôes nos dentes, nos tecidos moles da boca, além desgastar restauraçôes, em que pese a qualidade tecnológica dos atuais materiais restauradores empregados. A abrasão pode resultar do uso de qualquer agente polidor, tendo-se em consideração que a sua eficácia esta associada à dureza, ao tamanho e à forma das partículas (ANDRADE JÚNIOR e MACHADO, 2000). (1) No mercado brasileiro, os abrasivos mais usados nos dentifrícios são o carbonato de cálcio e a sílica, embora outros produtos similares possam estar presentes. (ANDRADE JÚNIOR; MACHADO, 2000; CURY, 2002)

O esmalte dental é, reconhecidamente, a estrutura biológica mineralizada mais resistente à abrasão, sendo pouco provável que a maioria dos dentifrícios venha a remover quantidades significativas desse tecido, desde que haja higidez do mesmo e que os procedimentos de escovação sejam tecnicamente adequados. $\mathrm{O}$ cemento é 35 vezes mais susceptível à abrasão do que o esmalte, enquanto que a dentina é 25 vezes mais susceptível. Embora protegidos pelo periodonto ou pelo esmalte, havendo algum comprometimento dessa proteção, tal como acontece em casos de recessão gengival, o risco de perda tecidual é muito grande, uma vez que essas estruturas não possuem o alto grau de mineralização que é encontrado no esmalte. (ANDRADE JÚNIOR;MACHADO, 2000)

Significativas reduções nos índices de cárie dental têm sido constatadas em vários países, atribuindo-se esse êxito à introdução dos dentifrícios fluoretados, dado que vem sendo comprovado através de relevantes estudos epidemiológicos. Esses resultados favoráveis estão diretamente associados à utilização do íon fluoreto, presente particularmente nos dentifrícios, uma vez que a regularidade do uso desses produtos, assegura importantes concentraçōes de fluoreto na interface placa-esmalte. (BOWEN,1995; CAMPOS, 2001; CURY, 2004; MARTHALER, 2004)

Os dentifrícios são importantes veículos de aplicação tópica de flúor, uma vez que encerram, em suas formulações, o fluoreto de sódio $(\mathrm{NaF})$, o monofluorfosfato de sódio (MFP), ou ainda a associação dessas duas substâncias, cujas concentraçōes variam em torno de 1.000 a 1.500 $\mathrm{ppmF}^{-}$, sendo indicadas concentrações mais baixas em cremes dentais infantis. (CAMPOS, 2001; CURY, 1989; CURY, 2002)

O monofluorfosfato de sódio (MFP) tem sido utilizado amplamente nas formulações de dentifrícios, principalmente devido à sua biocompatibilidade com os abrasivos que contêm cálcio, como, por exemplo, o fosfatodicálcio diidratado (DCPD) e o carbonato de cálcio $\left(\mathrm{CaCO}_{3}\right)$, permitindo que maior quantidade de flúor solúvel (ativo) se mantenha no produto, além da possibilidade de o cálcio dos sistemas abrasivos exercer um efeito aditivo ao MFP (KOO; CURY, 1999). O carbonato de cálcio, como abrasivo, reage com o fluoreto sob a forma de $\mathrm{NaF}$, diminuindo sua concentração ativa no dentifrício. Logo, com base nesse pressuposto, o MFP tem a sua indicação farmacológica no Brasil, ao se considerar que os dentifrícios populares contêm o íon cálcio. Potencialmente, 
esse íon é um agente capaz de inativar grande parte do flúor contido nos dentifrícios que encerram em suas formulações o NaF. (CURY, 2002)

Observações clínicas demonstraram que, quando incorporado aos dentifrícios com base de sílica, o fluoreto de sódio tem superioridade terapêutica em relação àqueles que contêm monofluorfosfato de sódio (MARKS et al., 1994; STEPHEN et al., 1994) Entretanto, outros estudos não demonstram essa superioridade; ao contrário, relatam que o efeito clínico da aplicação tópica de soluções de monofluorfosfato de sódio, se comparada com o fluoreto de sódio, é similar. (CRUZ, 1997)Assim, há de se considerar a importância da realização de investigaçôes científicas sobre a eficácia do íon fluoreto incorporado aos dentifrícios, visando não somente à prevenção da fluorose, principalmente em crianças, mas, principalmente, a possibilidade de controle da qualidade desses produtos comercializados em larga escala no Brasil, por razôes de seleção, custos e equivalência. Desse modo, o presente trabalho teve por objetivo estudar, in vitro, o grau de proteção do esmalte dental humano assegurado por essas duas formas de fluoreto, frente à queda do $\mathrm{pH}$.

\section{MATERIAIS E MÉTODOS}

$\mathrm{O}$ presente trabalho avaliou, in vitro, o grau de proteção do esmalte dental humano pelo íon fluoreto contido em sete dentifrícios-teste fluoretados, disponíveis comercialmente na cidade de Salvador, Bahia, Brasil: Colgate Proteção Anticáries $\left(\mathrm{DT}_{1}\right)$, Trends $\left(\mathrm{DT}_{2}\right)$, Prevent Antiplaca ( $\left.\mathrm{DT}_{3}\right)$, Colgate Antitártaro (DT4), Phillips 2 com Flúor ( $\mathrm{DT}_{5}$ ), Gessy Cristal Juá e Hortelã $\left(\mathrm{DT}_{6}\right)$ e Colgate Proteção Anticáries Gel $\left(\mathrm{DT}_{7}\right)$, comparativamente à ação do dentifrício controle, sem fluoreto, Phillips (DC). As taxas do cálcio e do fosfato liberados do esmalte tratado pelos dentifrícios e influenciados pela queda do $\mathrm{pH}$, foram determinadas por espectrometria de emissão atômica com plasma indutivamente acoplado (ICP-AES), taxas essas diretamente relacionadas ao grau de proteção do esmalte dentário conferido pelo íon fluoreto.

O Quadro 1 discrimina os dentifrícios estudados, utilizados no experimento, com os seus respectivos fabricantes, o tipo e a concentração de flúor presentes na sua formulação.

A extração e o isolamento do esmalte dental humano utilizados nas experimentações tiveram como referência a técnica de separação dos tecidos dentais, baseada na densidade dos mesmos (CAMPOS, 2001). O estudo da influ-

\begin{tabular}{|c|c|c|c|}
\hline Dentifrício & Fabricante & $\begin{array}{c}\text { Forma de } \\
\text { fluoreto }\end{array}$ & $\begin{array}{c}\text { Concentração } \\
\text { de fluoreto }\end{array}$ \\
\hline \multicolumn{4}{|l|}{ Dentifricio controle } \\
\hline DC (Phillips) & Sanofi Winthrop Farm. Ltda. & $\begin{array}{c}\text { Não } \\
\text { fluoretado }\end{array}$ & \\
\hline \multicolumn{4}{|l|}{ Dentifricios teste } \\
\hline DT $_{1}$ (Colgate Proteção Anticáries) & $\begin{array}{l}\text { Colgate - Palmolive, Divisão da } \\
\text { Kolynos do Brasil Ltda. }\end{array}$ & MFP & $1.500 \mathrm{ppmF}^{-}$ \\
\hline $\mathrm{DT}_{2}$ (Trends) & Kolynos do Brasil Ltda. & MFP & $1.000 \mathrm{ppmF}$ \\
\hline $\mathrm{DT}_{3}$ (Prevent Antiplaca) & Kolynos do Brasil Ltda. & MFP & $1.200 \mathrm{ppmF}$ \\
\hline $\mathrm{DT}_{4}$ (Colgate Antitártaro) & $\begin{array}{l}\text { Colgate - Palmolive Divisão da } \\
\text { Kolynos do Brasil Ltda. }\end{array}$ & $\mathrm{NaF}$ & $1.100 \mathrm{ppmF}$ \\
\hline $\mathrm{DT}_{5}$ (Phillips 2) & Sanofi Winthrop Farm. Ltda. & MFP & $1.200 \mathrm{ppmF}$ \\
\hline $\mathrm{TD}_{6}$ (Gessy Cristal) & Gessy Ltda. - Elisa Gibbs & MFP & $1.500 \mathrm{ppmF}$ \\
\hline $\mathrm{DT}_{7}$ (Colgate Proteção Anticáries Gel) & $\begin{array}{l}\text { Colgate - Palmolive, Divisão da } \\
\text { Kolynos do Brasil Ltda. }\end{array}$ & $\mathrm{NaF}$ & $1.500 \mathrm{ppmF}^{-}$ \\
\hline
\end{tabular}

Quadro 1- Dentifrícios estudados 
ência do íon fluoreto presente nos dentifrícios sobre o esmalte dental humano, submetido à variação do $\mathrm{pH}(\mathrm{pH} 4.5,5.5,6.0,6.8$ e 7.8), baseou-se na técnica preconizada por Tastaldi (1963), adaptada por Araújo (1996).

Através de espectrometria de emissão atômica, foram determinadas as taxas do cálcio e do fosfato liberados do esmalte tratado pelos dentifrícios e influenciados pela queda do $\mathrm{pH}$. A espectrometria se baseia na propriedade de átomos neutros ou íons monoatômicos em estado gasoso emitirem, quando térmica ou eletricamente excitados, radiações com comprimentos de onda característicos nas regiōes ultravioleta e visível. O conjunto de radiações emitidas por uma espécie constitui seu espectro de emissão, sendo que a avaliação dos comprimentos de onda permite a identificação dos elementos emissores, podendo a medida da intensidade das radiaçõos emitidas ser utilizada para determinar a concentração dos elementos presentes.

As taxas do cálcio e do fosfato total, liberados do esmalte previamente tratado pelos dentifrícios, são expressas em miligramas por decilitro, em função do tempo de 20 minutos ( $\mathrm{mg} \mathrm{Ca}^{2+} / \mathrm{dl} / 20 \mathrm{~min}$ ) ( TABELA 1; TABELA 2).

Os dados estão apresentados como médias e erros-padrão da média (EPM). As médias dos diversos grupos foram comparadas por análise de variância (One-way ANOVA -Teste de
Tukey, nível de significância 5\%) de medidas repetidas, levando-se em consideração a comparação entre o $\mathrm{pH}$ e as concentraçôes de cálcio e fosfato liberados do esmalte dental humano. Posteriormente, foi determinada correlação de Pearson entre as taxas do cálcio e do fosfato.

\section{RESULTADOS}

Os resultados estão descritos em função das taxas de cálcio e de fosfato determinadas a partir da liberação desses íons pelo esmalte dental humano tratado pelo dentifrício não fluoretado e pelos dentifrícios fluoretados, submetido aos valores de $\mathrm{pH} 4.5,5.5,6.0,6.8$ e 7.8 .

As Tabelas 1 e 2 e as Figuras 1 e 2 expressam, respectivamente, as taxas do cálcio e do fosfato liberados do esmalte dental previamente influenciado pela ação dos dentifrícios estudados e submetido aos diferentes níveis de $\mathrm{pH}$.

Ao se compararem as taxas do cálcio e do fosfato liberados do esmalte dental influenciado pelo dentifrício-controle (DC) e por cada dentifrício teste $\left(\mathrm{DT}_{1}, \mathrm{DT}_{2}, \mathrm{DT}_{3}, \mathrm{DT}_{4}, \mathrm{DT}_{5}\right.$, $\mathrm{DT}_{6}$ e $\mathrm{DT}_{7}$ ) e submetido à ação dos níveis de $\mathrm{pH} 4.5,5.5,6.0,6.8$ e 7.8, pode-se constatar ter havido diferenças estatísticas significativas em cada grupo estudado.

As mais baixas taxas do cálcio e do fosfato liberados do esmalte dental humano tratado

Tabela 1- Médias e EPM das taxas de cálcio ( $\mathrm{mg} / \mathrm{dl} / 20 \mathrm{~min}$ ) liberado do esmalte dental humano tratado por dentifrícios e submetido à variação do $\mathrm{pH}$ do meio

\begin{tabular}{|c|c|c|c|c|c|}
\hline Dentifrício & 7,8 & 6,8 & 6,0 & 5,5 & 4,5 \\
\hline \multicolumn{6}{|l|}{ Dentifrício-controle } \\
\hline DC (Phillips) & $7.590 \pm .152$ & $14.370 \pm .076$ & $11.130 \pm .026$ & $31.400 \pm .429$ & $125.500 \pm .645$ \\
\hline \multicolumn{6}{|l|}{ Dentifrícios-teste } \\
\hline $\mathrm{DT}_{1}$ (Colgate PA) & $5.240 \pm .279$ & $13.713 \pm .166$ & $14.230 \pm .082$ & $30.850 \pm .644$ & $124.800 \pm 1.031$ \\
\hline $\mathrm{DT}_{2}$ (Trends) & $3.340 \pm .199$ & $9.965 \pm .369$ & $12.730 \pm .163$ & $29.580 \pm .473$ & $126.000 \pm 1.225$ \\
\hline $\mathrm{DT}_{3}$ (Prevent) & $.350 \pm .007$ & $.953 \pm .031$ & $2.640 \pm .067$ & $18.280 \pm .733$ & $18.300 \pm .733$ \\
\hline $\mathrm{DT}_{4}$ (Colgate AT) & $.210 \pm .010$ & $.660 \pm .021$ & $2.290 \pm .149$ & $4.240 \pm .062$ & $15.900 \pm .107$ \\
\hline $\mathrm{DT}_{5}$ (Phillips 2) & $3.778 \pm .158$ & $9.753 \pm .513$ & $10.660 \pm .153$ & $26.400 \pm .260$ & $113.500 \pm 1.813$ \\
\hline $\mathrm{DT}_{6}$ (Gessy) & $5.438 \pm .120$ & $13.825 \pm .101$ & $13.660 \pm .067$ & $30.870 \pm .518$ & $123.700 \pm 1.071$ \\
\hline $\mathrm{DT}_{7}$ (Colgate Gel) & $.405 \pm .105$ & $.997 \pm .016$ & $3.010 \pm .024$ & $5.400 \pm .090$ & $17.500 \pm .366$ \\
\hline
\end{tabular}


Tabela 2 - Médias e EPM das taxas de fosfato $(\mathrm{mg} / \mathrm{dl} / 20 \mathrm{~min}$ ) liberado do esmalte dental humano tratado por dentifrícios e submetido à variação do $\mathrm{pH}$ do meio

\begin{tabular}{lccccc}
\hline \multicolumn{1}{c}{$\mathrm{pH}$} & 7,8 & 6,8 & 6,0 & \multicolumn{1}{c}{5,5} & \multicolumn{1}{c}{4,5} \\
\hline Dentifrício & & & & & \\
\hline Dentifŕ́cio-controle & & & & & \\
DC (Phillips) & $.353 \pm .037$ & $.455 \pm .046$ & $.085 \pm .015$ & $.365 \pm .027$ & $.818 \pm .191$ \\
Dentifrícios teste & & & & & \\
$\mathrm{DT}_{1}$ (Colgate PA) & $.410 \pm .020$ & $.455 \pm .011$ & $.580 \pm .0 .54$ & $.648 \pm .081$ & $1.225 \pm .068$ \\
$\mathrm{DT}_{2}$ (Tren ds) & $.488 \pm .059$ & $.420 \pm .033$ & $.560 \pm .055$ & $.508 \pm .028$ & $1.028 \pm .099$ \\
$\mathrm{DT}_{3}$ (Prevent) & $1.003 \pm .035$ & $1.633 \pm .094$ & $4.300 \pm .138$ & $7.840 \pm .104$ & $28.708 \pm .824$ \\
$\mathrm{DT}_{4}$ (Colgate AT) & $.665 \pm .058$ & $1.308 \pm .084$ & $3.152 \pm .070$ & $6.713 \pm .119$ & $25.338 \pm .166$ \\
$\mathrm{DT}_{5}$ (Phillips 2) & $.888 \pm .039$ & $.875 \pm .041$ & $.355 \pm .013$ & $.722 \pm .011$ & $1.663 \pm .163$ \\
$\mathrm{DT}_{6}$ (Gessy) & $.468 \pm .028$ & $.515 \pm .018$ & $.475 \pm .006$ & $.658 \pm .029$ & $1.383 \pm .825$ \\
$\mathrm{DT}_{7}$ (Colgate Gel) & $.502 \pm .086$ & $1.415 \pm .044$ & $4.042 \pm .036$ & $8.453 \pm .152$ & $27.155 \pm .350$ \\
\hline
\end{tabular}

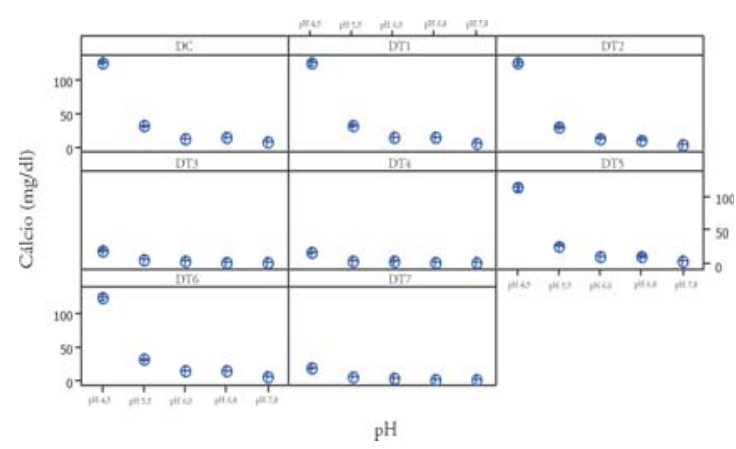

Figura 1 - Taxas do cálcio liberado do esmalte dental humano tratado por dentifrícios e submetido aos $\mathrm{pH} 4.5,5.5$, $6.0,6.8$ e 7.8 .

tanto pelo dentifrício-controle quanto pelos sete dentifrícios-teste foram detectadas após se submeter essa estrutura biológica à ação do $\mathrm{pH} 7,8$ e revelaram diferenças estatísticas significativas ao se compararem os resultados obtidos pela ação dos dentifrícios-teste com as taxas resultantes da ação do dentifrício-controle.

As taxas de cálcio mais baixas em $\mathrm{pH} 7,8$ ficaram por conta do esmalte tratado pelos dentifrícios fluoretados $\mathrm{DT}_{3}, \mathrm{DT}_{4}$ e $\mathrm{DT}_{7}(.350$ $\pm .007, .210 \pm .010$ e $.405 \pm .105$, respectivamente). Por outro lado, a taxa de fosfato mais alta obtida em $\mathrm{pH}$ 7,8 ficou por conta do esmalte influenciado pelo dentifrício fluoretado $\mathrm{DT}_{3}$.

Nos demais níveis de $\mathrm{pH}(4.5,5.5,6.0$ e 6.8), os dentifrícios fluoretados $\mathrm{DT}_{3}, \mathrm{DT}_{4} \mathrm{e}$ $\mathrm{DT}_{7}$ respondem pelas menores taxas de cálcio liberado do tecido tratado e pelas maiores taxas de fosfato liberado do esmalte dental..

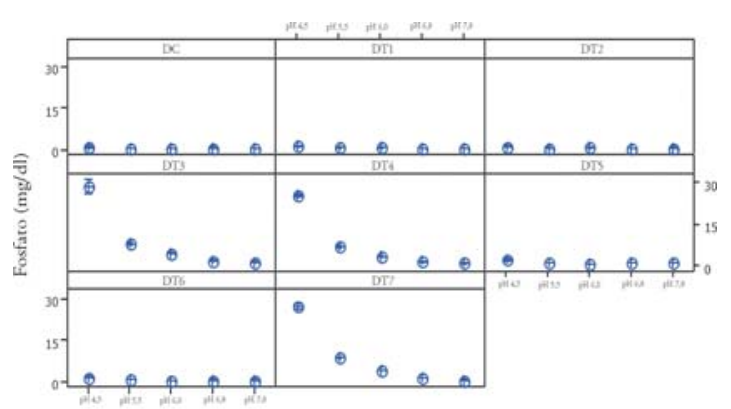

$$
\mathrm{pH}
$$

Figura 2 - Taxas do fosfato liberado do esmalte dentário humano tratado por dentifrícios e submetido aos $\mathrm{pH} 4.5,5.5$, $6.0,6.8$ e 7.8 .

A Figura. 3 expressa a correlação entre as taxas de cálcio e de fosfato nos níveis de $\mathrm{pH} 4.5$, 5.5, 6.0, 6.8 e 7.8, sendo essa correlação negativa e estatisticamente significativa para todas

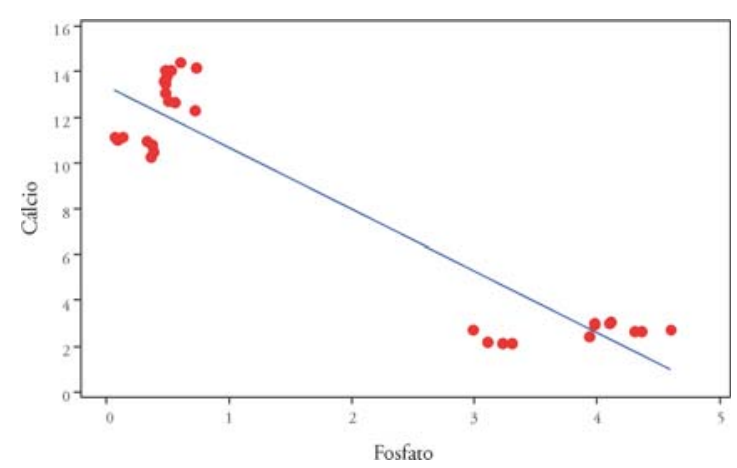

Figura 3 - Correlação das taxas do cálcio e do fosfato liberados do esmalte dental humano tratado por dentifrícios e submetido aos $\mathrm{pH} 4.5,5.5,6.0,6.8$ e 7.8 . 
os níveis de $\mathrm{pH}$, exceto para o $\mathrm{pH} 7.8$, para o qual não foi detectada significância alguma.

\section{DISCUSSÃO}

Os dentifrícios analisados demonstraram diferenças tanto em relação ao tipo de flúor presente em suas formulações - fluoreto de sódio $(\mathrm{NaF})$ e monofluorfosfato de sódio (MFP) quanto na concentração desse íon, ou seja:

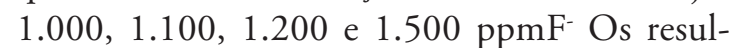
tados da presente investigação revelaram a eficácia do íon fluoreto em promover, através do seu efeito residual, proteção ao esmalte dental humano submetido aos níveis de $\mathrm{pH} 7.8,6.8$, 6.0, 5.5 e 4.5 .

A ação protetora do íon fluoreto contido nos dentifrícios registrada por Cury (2004) e Marthaler (2004 foi confirmada através deste estudo, realizado in vitro, mediante o decréscimo das taxas do cálcio e do fosfato total liberados do esmalte dentário humano submetido ao meio ácido, o que se deveu ao princípio que considera que a presença do fluoreto nas soluçōes ácidas subsaturantes de cálcio e de fosfato diminui a solubilidade do esmalte, pois, mesmo em $\mathrm{pH}$ ácido, a presença desse elemento compensará, parcialmente, a perda de cálcio e de fosfato sob a forma de hidroxiapatita, pelo ganho de cálcio, fosfato e fluoreto, em virtude da manutenção do meio supersaturado em relação ao produto de solubilidade da fluorhidroxiapatita. (CURY, 1989)

Estudos realizados, in vitro e in vivo, em animais de laboratório, apresentaram consistentes resultados clínicos favoráveis ao fluoreto de sódio em relação ao monofluorfosfato de sódio, em que pese alguns ensaios clínicos com dentifrícios que contêm o fluoreto de sódio ou o monofluorfosfato de sódio terem demonstrado substanciais reduções de lesões incipientes de esmalte, muito próximas. Estudos que comparam os efeitos de dentifrícios contendo fluoreto de sódio com cremes dentais que encerram em sua formulação o monofluorfosfato de sódio revelaram que os primeiros são invariavelmente superiores àqueles com monofluorfosfato de sódio, e ensaios que usam modelos intra-orais em humanos têm comprovado que, em lesões incipientes de esmalte, os dentifrícios com fluoreto de sódio resultam em deposições de fluoreto de cálcio significativamente maiores do que com o monofluorfosfato de sódio (BOWEN, 1995) A análise da ação dos sete dentifríciosteste selecionados para o presente estudo, realizado in vitro, concluiu pela maior proteção do esmalte dentário pelo fluoreto de sódio, em comparação com o monofluorfosfato de sódio.

Possivelmente, as taxas de cálcio e de fosfato liberados do esmalte tratado pelo dentifrício controle, DC, não foram maiores em virtude da elevação do $\mathrm{pH}$ causado por esse produto, uma vez que o mesmo detém um $\mathrm{pH}$ extremamente alcalino (10.37). Dessa forma, o dentifrício-controle contribuiu, efetivamente, para a alcalinidade do meio, reduzindo ainda mais a possibilidade de alguma perda de cálcio e de fosfato do esmalte dentário submetido ao $\mathrm{pH} 7,8$. Essa interferência protetora foi sendo reduzida, gradativamente, à medida que se impôs a queda do $\mathrm{pH}$ do meio ao esmalte dentário, conforme está expresso nas Figuras1 e 2.

Os dentifrícios fluoretados $\mathrm{DT}_{3}, \mathrm{DT}_{4} \mathrm{e}$ $\mathrm{DT}_{7}$ analisados a partir das taxas do cálcio liberado do esmalte, foram os que efetivamente protegeram o esmalte dentário humano in vitro. As baixas taxas do cálcio que foram liberadas do esmalte dentário por influência desses produtos revelaram diferenças estatisticamente significativas, em relação ao dentifrício-controle. Esse fenômeno resultou, provavelmente, da presença do fluoreto de sódio nos dentifrícios fluoretados $\mathrm{DT}_{4}$ e $\mathrm{DT}_{7}$ o que favoreceu, possivelmente, a formação do fluoreto de cálcio, não havendo assim a liberação desse íon para o meio, e do monofluorfosfato de sódio contido no dentifrício teste $\mathrm{DT}_{3}$, que, quando em solução, apresentou-se sob a forma de uma solução de $\mathrm{pH}$ 5,93, ou seja, o $\mathrm{pH}$ mais baixo entre todos os dentifrícios estudados, o que deve ter favorecido a formação do fluoreto de cálcio. Essa proteção da desmineralização, fornecida pelo dentifrícioteste $\mathrm{DT}_{3}$ decorreu, provavelmente, do valor de pH 5.93 apresentado, enquanto que, nos demais produtos com monofluorfosfato, os níveis de $\mathrm{pH}$, se situaram acima de 8,0 , e não propiciaram esse fenômeno de forma tão efetiva. Quan- 
do comparados aos produtos $\mathrm{DT}_{4} \mathrm{e} \mathrm{DT}_{7}$ todos os demais dentifrícios fluoretados, exceto o $\mathrm{DT}_{3}$, apresentaram menor proteção, fato que provavelmente terá decorrido da presença do fluoreto apenas sob a forma de monofluorfosfato de sódio em suas formulações.

Por outro lado, é possível se verificar, na Tabela 2, que os dentifrícios fluoretados $\mathrm{DT}_{3}$, $\mathrm{DT}_{4}$ e $\mathrm{DT}_{7}$, quando analisados a partir das taxas do fosfato liberado do esmalte, foram os que revelaram as taxas mais altas desse elemento. Esse resultado, provavelmente, decorreu do fato de o dentifrício $\mathrm{DT}_{4}$ apresentar, em sua formulação, o pirofosfato de cálcio, como agente inibidor da formação do cálculo dental, e os dentifrícios fluoretados $\mathrm{DT}_{3}$ e $\mathrm{DT}_{7}$ em função de conterem o flúor sob a forma de $\mathrm{NaF}$, apresentarem a sílica como agente abrasivo principal. De acordo com Andrade Júnior e Machado (2000), os dentifrícios que contêm sílica geralmente são de alta abrasividade, apresentando partículas de tamanhos irregulares e com resistência aumentada à pressão das cerdas da escova dentária, contrariamente aos materiais mais porosos, como o carbonato de cálcio, de menor abrasividade.

A análise dos resultados permite, ainda, estabelecer uma correlação entre a eficácia dos dentifrícios e a concentração de flúor, ou seja, os testes realizados com os dentifrícios que contêm monofluorfosfato de sódio na concentração de 1.000 e 1.200 ppmF- $^{-}\left(\mathrm{DT}_{2}\right.$ e $\left.\mathrm{DT}_{5}\right)$ indicam uma maior proteção sobre o esmalte dentário em relação àqueles que possuem esse mesmo componente na concentração de $1.500 \mathrm{ppmF}^{-}$ $\left(\mathrm{DT}_{1}\right.$ e $\left.\mathrm{DT}_{6}\right)$. Tal constatação permite que se assegurem os benefícios do íon fluoreto com doses menores desse elemento, prevenindo, inclusive, a possibilidade de fluorose em crianças, sujeitas à ingestão de dentifrícios por falhas de vigilância de seus pais ou responsáveis.

Os resultados decorrentes dessas experimentações estão de acordo com os achados dos estudos comparativos entre o NaF e o MFP, realizados por Morganstein (1994), que conclui pela superioridade do $\mathrm{NaF}$, assegurando aos indivíduos que fazem uso regular de dentifrícios com esa forma ativa de flúor uma menor necessidade de tratamento odontológico, uma vez que as possíveis lesões de esmalte são remineralizadas precocemente.
Faller e colaboradores (1997), ao testarem a eficácia anticárie de 20 dentifrícios disponíveis para consumo na China, através da aplicação de testes de captação de fluoreto pelo esmalte dentário humano desmineralizado, da avaliação de alterações da microdureza superficial do esmalte em função de cada dentifrício testado, da medida de resistência subseqüente, após submeter essa estrutura dentária ao desafio ácido, além da análise quantitativa por microrradiografia do teor de esmalte remineralizado, concluíram que os dentifrícios que contêm fluoreto de sódio e fluoreto estanhoso, em sílica, foram os mais eficazes. Além disso, ao avaliarem a incorporação do fluoreto ao esmalte desmineralizado, comprovaram que o monofluorfosfato de sódio foi menos efetivo do que o fluoreto de sódio.

O presente trabalho, realizado in vitro, demonstrou a maior eficácia protetora dos dentifrícios que contêm fluoreto de sódio $(\mathrm{NaF})$ sobre o esmalte dentário humano ao serem comparados aos demais dentifrícios, que encerram o monofluorfosfato de sódio (MFP). Essa ação deveu-se, possivelmente, à ocorrência do íon fluoreto livre e totalmente aproveitável sob aquela forma. Os resultados encontrados estão de acordo com as conclusões de Bowen (1995), que levam em conta dados in vitro e in situ, estudos com roedores, estudos farmacocinéticos em humanos e ensaios clínicos controlados, de que os dentifrícios adequadamente formulados que contêm $\mathrm{NaF}$ são mais efetivos do que aqueles em que está presente o MFP na mesma concentração, e essa diferença, quanto ao efeito, é clinicamente relevante. Ainda de acordo com esse autor, o MFP não possui a mesma biodisponibilidade que o fluoreto de sódio e, na ausência das fosfatases, num sistema in vitro, o monofluorfosfato de sódio liberta o íon fluoreto muito lentamente, não revelando, portanto, uma efetividade comparável à demonstrada pelo fluoreto de sódio.

Tais constatações concordam com as observações de Stephen e colaboradores (1994), que estudaram os efeitos de dentifrícios com fluoreto de sódio, monofluorfosfato de sódio e a associação de fluoreto de sódio com 
trimetafosfato, utilizados durante três anos por adolescentes escoceses, sobre o incremento de cárie. Esses autores concluíram que o aumento médio do CPOS, após três anos, em indivíduos que usavam dentifrícios com apenas o fluoreto de sódio, foi $6,4 \%$ mais baixo em relação ao dos que usavam dentifrício com monofluorfosfato, e que a diferença entre os indivíduos tratados pelo produto com a associação fluoreto de sódio/ trimetafosfato de sódio e os tratados com o monofluorfosfato de sódio foi da ordem de $8,1 \%$. Esses achados levaram os autores a afirmarem que o fluoreto de sódio tem comprovada superioridade sobre o monofluorfosfato, quando incorporado aos dentifrícios bem formulados, com base de sílica.

A partir do final da década de 80 , houve uma melhoria qualiquantitativa em termos de dentifrícios fluoretados no Brasil. De acordo com uma Resolução específica do Ministério da Saúde, os dentifrícios deveriam conter de 1.000 a $1.500 \mathrm{ppm}$ de flúor solúvel (ativo), iônico ou ionizável. Lamentavelmente essa Resolução foi modificada em 1994, quando o termo "solúvel" foi retirado. Assim, a atual Portaria eliminou do texto da anterior a exigência de o flúor estar solúvel na formulação. Isso possibilita que um fabricante possa lançar no mercado um dentifrício com até $1.500 \mathrm{ppm}$ de flúor tendo mais de $50 \%$ do flúor inativo para controlar a cárie dental. (CURY, 2002; CURY, 2004)

Assim, é extremamente importante a continuidade de novas experimentações com vistas a subsidiar, cada vez mais, os dados da literatura científica que tratam da eficácia protetora do fluoreto de sódio, do monofluorfosfato de sódio e da associação entre esses dois agentes, em face da importância de se obterem respostas preventivas e terapêuticas cada vez mais eficazes, com utilização de baixas concentrações de fluoreto, bem como contribuir para o adequado controle da qualidade industrial desses produtos.

\section{CONCLUSÕES}

De acordo com os experimentos realizados e aqui apresentados, pode-se concluir que:

a) os dentifrícios que contêm MFP (DT ${ }_{1}$, $\mathrm{DT}_{2}, \mathrm{DT}_{3}, \mathrm{DT}_{5}$ e $\mathrm{DT}_{6}$ ) parecem induzir a proteção do esmalte dentário humano em $\mathrm{pH}$ $7,8,6,8$ e 6,0 , quando comparados com o dentifrício-controle (DC) não fluoretado;

b) o dentifrício que contém MFP a 1.200 $\operatorname{ppmF}^{-}\left(\mathrm{DT}_{3}\right)$ parece induzir a proteção do esmalte dentário humano frente aos valores de $\mathrm{pH}$ $7,8,6,8,6,0,5,5,4,5$, ao ser comparado com o dentifrício-controle; (DC), contudo essa proteção é menos efetiva nos valores de $\mathrm{pH}$ abaixo do nível considerado crítico para o esmalte dental humano ( $\mathrm{pH} 5,5$ e 4,5);

c) os dentifrícios-teste com $\mathrm{NaF}$ em concentraçōes entre $1.000 \mathrm{ppmF}^{-}$e $1.200 \mathrm{ppmF}^{-}$ parecem induzir a proteção do esmalte dentário humano com mais intensidade, uma vez comparados aos que contêm MFP em concentrações mais altas - 1.500 ppmF;

d) é possível identificar uma correlação negativa, estatisticamente significativa, entre as taxas do cálcio e do fosfato liberados do esmalte dental humano nos níveis de $\mathrm{pH} 4.5,5.5,6.0 \mathrm{e}$ 6.8 , ainda que protegido pelo fluoreto; entretanto, no $\mathrm{pH} 7,8$, essa correlação não foi estatisticamente significativa.

\section{Quantitative analysis of the demineralization of the dental enamel submitted to the action of fluorinated dentifrices}

\section{Abstract}

The dental caries is a multifactorial illness, characterized as a dynamic process, originated from the disequilibrium in the phenomena of demineralization and re-mineralization. Several studies have demonstrated that the prevalence of caries in the majority of the countries have diminished in last the two decades due to implantation of public health programs, to the use of fluorine through the fluoridate water supplying and the consumption of fluoridate dentifrices. The fluoride contained in the dentifrices is capable to 
reduce the mineral loss of the enamel of teeth and to activate the ionic replacement in units with white spots injuries and incipient injuries of caries. This data justify the present study that aimed to determine, in vitro, the human dental enamel protection degree by fluoride; in the forms of sodium fluoride (NaF) and sodium mono-fluoride-phosphate of (MFP), contained in seven dentifrice-test (Colgate Anti-caries Protection (DT1), Trends (DT2), Prevent Anti-placa (DT3), Colgate Anti-tártaro (DT4), Phillips 2 with Fluorine (DT5), Gessy Crystal Juá and Mint (DT6) and Colgate Anti-caries Protection Gel (DT7)) comparativily to the action of the control-dentifrice, without fluoride, Phillips (DC), due to its $p H$ fall to critical levels. The taxes of calcium and phosphate released from the enamel, previously treated by the dentifrices, which had been determined through the technique of spectrometry of atomic emission with plasma connected inductively. The findings had shown the protective power of these products, indicating the appropriate control by the industrial quality. It was also evidenced, greater protection of the enamel for the dentifrices containing the fluoride of sodium in relation to those containing monofluorphosphate of sodium and a negative correlation between the taxes of calcium and phosphate released from the human enamel.

Keywords: Dental enamel - dentifrices; sodium fluoride (NaF); sodium mono-fluoride-phosphate (MFP)

\section{REFERÊNCIAS}

ANDRADE JÚNIOR, A.; MACHADO, W.A.S. Caracterização físico-química doscomponentes inorgânicos dos dentifrícios. R. ABOPREV, Rio de Janeiro, v.3, n.2, p.50-56, 2000 .

ARAÚJO, R.P.C. Interferência do íon fluoreto na solubilidade do esmalte dentário humano: ação de dentifrícios e enxaguatórios. 1996. 143f. il. Tese (Livre Docência em Odontologia) - Faculdade de Odontologia, Universidade Gama Filho, Rio de Janeiro, 1996.

BOWEN, W.H. The role of fluoride toothpastes in prevention of dental caries. J. Royal Soc. Med., London, v.88, p.505-507, 1995.

CAMPOS, E.J. Influência do íon fluoreto no esmalte dentário humano: análise por espectrometria de emissão atômica. 2001. 129p. Dissertação (Mestrado em Clínica Odontológica) - Faculdade de Odontologia, Universidade Federal da Bahia, Salvador, 2001.

CRUZ, R.A. Considerações clínicas e laboratoriais sobre a reatividade de compostos fluoretados aplicados topicamente no esmalte dental humano. In: KRIGER, L. (Coord.) Promoção de saúde bucal. São Paulo: Artes Médi- cas: ABOPREV 1997. cap. 9, p.168-193.

CURY, J.A. . Dentifrícios: como escolher e como indicar. In: ODONTOLOGIA. São Paulo: APCD 2002. v.4, p.281-295.

CURY, J.A. Uso do flúor. In: BARATIERI, L.N. Dentística: procedimentos preventivos e restauradores. 2.ed. São Paulo: Santos, 1989. p.43-67.

CURY, J.A. et al. The importance of fluoride dentifrices to the current dental caries prevalence in Brazil. Braz. Dent. J., Ribeirão Preto, v.15, n.3, p.167-174, 2004.

FALLER, R.V. et al. The comparative anticaries efficacy of Crest toothpaste relative to some marketed chinese toothpastes:results of in vitro $\mathrm{pH}$ cycling testing. Int. Dent. J., London, v.47, p.313-320, Dec. 1997.

KOO, H.; CURY, J.A. Avaliação in situ de um dentifrício contendo MFP/DCPD na incorporação de flúor e remineralização do esmalte dental humano. R. Odontol. Univ. São Paulo, São Paulo, v.13, n.3, jul./set., p.245-249, 1999.

MARKS, R.G. et al. Results from a three-years caries clinical trial comparing $\mathrm{NaF}$ and SMFP 
fluoride formulations. Int. Dent. J., London, v.44, n.3, p.275-285, 1994. Suppl.1.

MARTHALER, T.M. Changes in dental caries 1953-2003. Caries Res., Basel, v.38, p.173$181,2004$.

MORGANSTEIN, S.I. Clinical results: implications for prevention and treatment in general dental practice. Int. Dent. J., London, v.44, n.3, p.297-299, June 1994.
PALOMO, F. et al. The effect of a dentifrice containing triclosan and copolymer on plaque formation and gingivitis: a 14-week clinical study. Am. J. Dent., San Antonio, v.2, p.231237, 1989.

STEPHEN, K.W. et al. The effect of $\mathrm{NaF}$ and SMFP toothpastes on three-years caries increments in adolescents. Int. Dent. J., London, v.44, n.3 , p.287-295, 1994. Suppl.1.

\section{Agradecimentos} dados.

À Professora Dra. Maria Cristina Teixeira Cangussu, pelo auxílio na análise estatística dos

Recebido em / Received: 26/10/2005

Aceito em / Accepted: 15/12/2005 